\title{
SEA OIL SEEP MONITORING USING A TIME SERIES OF CO-POLARIZED COHERENT SAR MEASUREMENTS
}

\author{
Carina R. de Macedo ${ }^{1}$, Andrea Buono ${ }^{1}$, Ferdinando Nunziata ${ }^{1}$, Domenico Velotto ${ }^{2}$, Maurizio Migliaccio ${ }^{1}$ \\ ${ }^{1}$ Università di Napoli Parthenope, Dipartimento di Ingegneria 80143 Napoli, Italy \\ ${ }^{2}$ German Aerospace Center (DLR), Remote Sensing Technology Institute, 28199 Bremen, Germany
}

\begin{abstract}
In this study, coherent co-polarized synthetic aperture radar (SAR) information is exploited for sea oil seep monitoring purposes. A time series of 33 TerraSAR-X StripMap SAR data, collected over a well-known oil seep in Gulf of Mexico, is exploited to analyze the influence of incidence angle and wind conditions on the co-polarized phase difference (CPD). Furthermore, the benefits of using phase information for oil seep observation are assessed. Experimental results show that: i) incidence angle has a remarkable effect in broadening/shrinking the CPD statistical distribution and, ii) the CPD provides spatial information on the oil seep damping properties.
\end{abstract}

Index Terms - oil seep, synthetic aperture radar, polarimetry, co-polarized phase difference.

\section{INTRODUCTION}

Oil seeps are the natural low-rate flow of liquid or gaseous crude oil and tar from the bottom of the oceans. They have an ecological and economical importance since they increase phytoplankton and carbon productivity regionally and provide sites of potential resources [1]. Oil seeps manifest themselves as a random flow of oil droplets that vary in size, rate, concentration and shape. This flow is affected by underwater currents and mass movements inside the water column and by surface currents, wind and other meteo-marine processes on the sea surface. The latter also expose the oil surface layer to aging and weathering processes [2].

Hence, sea oil seep monitoring is a challenging task for which synthetic aperture radar (SAR) is a key cost-effective tool being an almost all-weather and fine spatial resolution imaging sensor [3]. In principle, sea oil slicks can be observed by means of SAR since they attenuate the short Bragg resonant waves responsible of the sea surface backscattering and, therefore, they appear in conventional gray-tones SAR imagery as homogeneous areas darker than the background sea.

This study is partially supported by Università degli Studi di Napoli Parthenope under the project ID DING 202 and by European Space Agency under the Dragon-4 project ID 32235.
Several studies exploited single-polarization intensity information to observe natural oil seeps achieving satisfactory results in discriminating between oil seeps and accidental/deliberated oil discharges $[4,5]$. Nevertheless, the intensity information alone is not enough to infer the chemical and physical oil properties [2,3]. Within this context, there is a wide consensus in literature on the extra-benefits provided by co-polarized phase information in both performing a rough estimation of the spatial variability of the oil damping properties and distinguishing oil slicks from weak-damping look-alikes $[2,3,6]$.

In this study, a time series of 33 coherent co-polarized TerraSAR-X (TSX) SAR measurements has been exploited to observe a well-known oil seep in Gulf of Mexico. We investigated the influence of angle of incidence (AOI), noise equivalent sigma zero (NESZ) and sea state conditions on the co-polarized phase information evaluated over both slickfree and slick-covered sea surface and, furthermore, we assessed the dual-polarimetric SAR capabilities to provide deeper information regarding the spatial/temporal variability of the sea oil seep damping properties.

The paper is organized as follows: in section 2, the polarimetric model for interpreting coherent dual-polarimetric information is presented; in section 3 the dataset is described, while in section 4 experiments are designed and discussed; conclusions are drawn in section 5 .

\section{POLARIMETRIC MODEL}

The X-Bragg scattering model, adopted in this paper to describe the sea surface polarimetric scattering, is an extended version of the Bragg scattering theory that takes into account the non-negligible cross-polarized return and the depolarizing effects. This model was first described in [7] to model slightly rough surface backscattering and, in [8], it was specialized for the sea surface backscattering. Note that, since in this paper we are interested on the exploitation of copolarized information for sea oil seep detection purposes, the way the X-Bragg model introduces cross-polarized backscattering does not affect our analysis. Nevertheless, to adopt the X-Bragg sea surface scattering model allows us relating the co-polarized information to the sea state conditions 


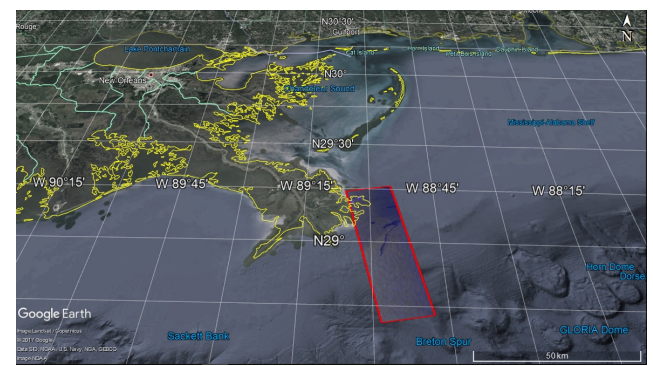

Fig. 1. Map of the study area in Gulf of Mexico, where the TSX SAR imagery show the oil seep signatures as patches darker than the background sea.

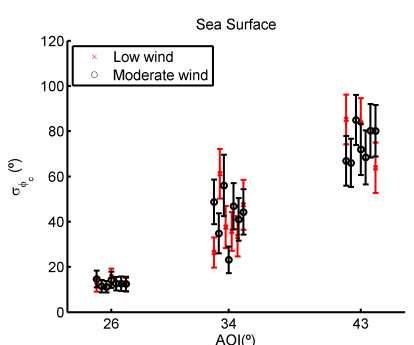

(a)

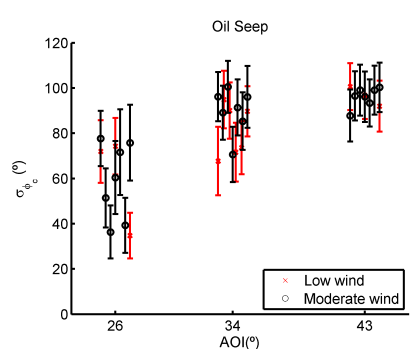

(b)
Fig. 2. Behavior of $\sigma_{\mathrm{CPD}}$ with respect to AOI over: (a) slickfree and (b) slick-covered sea surface. Data-points belonging to low and moderate wind regimes are shown in red and black, respectively.

through the tilting angle. Within this context, using the $\mathrm{X}-$ Bragg scattering model and considering intermediate AOI, the covariance matrix can be predicted over sea surface as follows [8]:

$$
\mathbf{C}=\mathbf{U T U}^{-\mathbf{1}}
$$

where $\mathbf{U}$ is a linear "con-similarity" transformation and $\mathbf{T}$ is the coherence matrix predicted according to the $\mathrm{X}-\mathrm{Bragg}$ model [8]:

$$
\mathbf{T}=\left(\begin{array}{ccc}
|\gamma|^{2} & \gamma \alpha \operatorname{sinc}(2 \beta) & 0 \\
\gamma^{*} \alpha^{*} \operatorname{sinc}(2 \beta) & \frac{1}{2}|\delta|^{2}(1+\operatorname{sinc}(4 \beta)) & 0 \\
0 & 0 & \frac{1}{2}|\delta|^{2}(1-\operatorname{sinc}(4 \beta))
\end{array}\right)
$$

where $\gamma=R_{H}+R_{V}, \alpha=R_{H}^{*}+R_{V}^{*}$ and $\delta=R_{H}-R_{V}$. $R_{H}$ and $R_{V}$ are AOI-dependent Bragg scattering coefficients for horizontal and vertical components, respectively, that take into account the dielectric properties of sea surface, while $\beta$ is the width of the uniform distribution assumed to statistically model the local surface tilting [7].

Once the covariance matrix that characterize sea surface backscattering is obtain, the co-polarized phase difference (CPD) can be derived which was successfully and widely used for sea oil slick observation purposes [3,6]:

$\mathrm{CPD}=\angle C(1,3)=\angle\left(\frac{\alpha^{2}}{2}-\frac{\delta^{2}}{4}(1-\operatorname{sinc}(4 \beta))-j \Im(\gamma \alpha \operatorname{sinc}(2 \beta))\right)_{(3)}$

The terms $\angle$ and $\Im$ mean phase and imaginary part, respectively. According the Eq. (3), the CPD depends on the sensor's imaging parameters (i.e., AOI and incident wavelength) and the surface properties ( $\beta$ and dielectric permittivity).

In [6], it was shown that mean and standard deviation of the CPD distribution (respectively, $\mu_{C P D}$ and $\sigma_{C P D}$ ) are related to the scattering properties of the observed surface. The analytical expression of the CPD distribution, given in [6], is shown in eq. (4) on the top of the next page, where $\mathrm{N}$ is the number of looks and $\rho$ is the correlation of the copolarized channels, respectively. $L(\cdot)$ and $\Gamma(\cdot)$ are the first kind-Legendre and Gamma functions, respectively. The width of $p_{\mathrm{CPD}}, \sigma_{\mathrm{CPD}}$, is considered as a measure of the degree of correlation between the co-polarized channels. When they are totally correlated, $\sigma_{\mathrm{CPD}}$ tends to $0^{\circ}$; when they are completely uncorrelated, $\sigma_{\mathrm{CPD}}$ is such that the CPD distribution approaches a uniform distribution. Since the slick-free sea surface is characterized by an almost deterministic Bragg scattering mechanism, the high degree of correlation between the co-polarized channels results into lower values of $\sigma_{\mathrm{CPD}}$, while the oil slick-covered sea surface is characterized by larger $\sigma_{\mathrm{CPD}}$ values due to the depolarizing nature of oil seep backscattering [6].

\section{SAR DATASET AND STUDY AREA}

The study area is in the northen part of Gulf of Mexico near the Mississippi river delta (see Fig. 1). Satellite imagery collected by TSX mission observed an almost persistent oil seep related to the leaking of the Taylor Energy oil platform, destroyed in 2004 during the Hurricane Ivan. Although this feature may not be considered a natural oil seep strictly, it is an unprecedented opportunity to exploit a statistically reliable polarimetric SAR dataset. In this study a time series of 33 coherent HH-VV TSX SAR data acquired between July 2011 and April 2016 in StripMap mode is considered, which was collected under low-to-moderate wind conditions (wind speed in the range $1.5-8 \mathrm{~m} / \mathrm{s}$ ) at intermediate AOIs (range $24^{\circ}-$ $44^{\circ}$ ). Wind speed information is provided by buoy data freely available from the National Data Buoy Center of the National Oceanic and Atmospheric Administrations (NOAA).

\section{EXPERIMENTAL RESULTS}

In the first experiment, the behavior of $\sigma_{\mathrm{CPD}}$ over both slickfree and slick-covered sea surface is investigated with respect to acquisition parameters (NESZ and AOI) and meteo-marine conditions (wind regime). The SAR imagery were partitioned into 5 subsets according to AOI and wind regime. According 


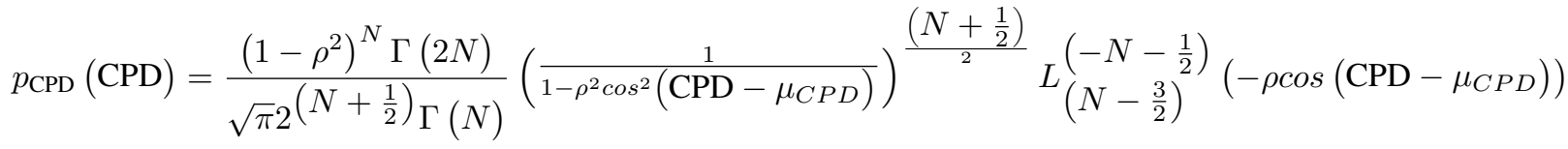

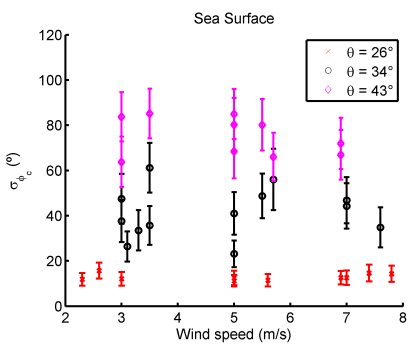

(a)

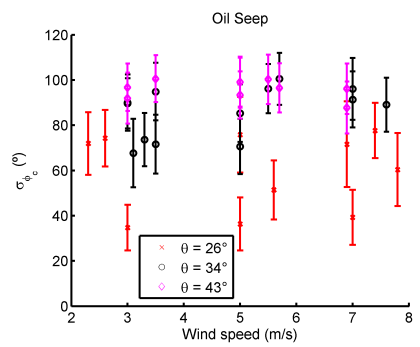

(b)
Fig. 3. Behavior of $\sigma_{\mathrm{CPD}}$ with respect to wind speed over: (a) slick-free and (b) slick-covered sea surface.

Table 1. SNR VALUES FOR THE SAR DATASET PARTIONED ACCCORDING AOI.

\begin{tabular}{|c|c|c|c|c|c|c|}
\hline AOI $\left({ }^{\circ}\right)$ & \multicolumn{2}{|c|}{26} & \multicolumn{2}{c|}{34} & \multicolumn{2}{c|}{43} \\
\hline ROI & Slick-free & Oil seep & Slick-free & Oil seep & Slick-free & Oil seep \\
\hline SNR & 12.6 & 4.4 & 5.4 & 0.1 & 2.1 & -0.4 \\
\hline
\end{tabular}

to AOI, the SAR scenes are classified as low $\left(26^{\circ}\right)$, intermediate $\left(34^{\circ}\right)$ and large $\left(43^{\circ}\right)$; while, according to wind regime, they are grouped as low (wind speed $\leq 3.5 \mathrm{~m} / \mathrm{s}$ ) and moderate (wind speed $\geq 5 \mathrm{~m} / \mathrm{s}$ ).

$\sigma_{\mathrm{CPD}}$ is evaluated using a $9 \times 9$ moving window and, then, 1000 pixels belonging to two regions of interest (ROIs), i.e., sea surface and oil seep, are randomly sampled for each SAR scene. In Fig. 2 and Fig. 3, the mean and the standard deviation values of $\sigma_{\mathrm{CPD}}$ derived over both ROIs are shown versus AOI (for low and moderate wind regimes, depicted in red and black errorbars, respectively) and wind speed (for low, intermediate and large AOI, depicted in red, black and magenta errorbars, respectively), respectively.

In Fig. 2, considering the sea surface (Fig. 2(a)), $\sigma_{\mathrm{CPD}}$ almost linearly increases when increasing AOI. When low wind regime applies, $\sigma_{\mathrm{CPD}}$ values increase of $275 \%$ (65\%) when AOI moves from $26^{\circ}\left(34^{\circ}\right)$ to $34^{\circ}\left(43^{\circ}\right)$ in average; while, under moderate regime, $\sigma_{\mathrm{CPD}}$ values, in average, increase of $173 \%$ (109\%) when AOI increases from $26^{\circ}\left(34^{\circ}\right)$ to $34^{\circ}$ $\left(43^{\circ}\right)$. Considering the oil seep (Fig. 2(b)), the increasing trend of $\sigma_{\mathrm{CPD}}$ with $\mathrm{AOI}$ is less pronounced than in the sea surface case, i.e., under low wind conditions, $\sigma_{\mathrm{CPD}}$ values increase from $24 \%(5 \%)$ when AOI increases from $26^{\circ}\left(34^{\circ}\right)$ to $34^{\circ}\left(43^{\circ}\right)$, in average; while, when moderate wind regime applies, it is stronger, i.e., $52 \%(20 \%)$.

In Fig. 3(a) it can be noted that, over slick-free sea surface ROI, the increasing of wind speed does not affect $\sigma_{\mathrm{CPD}}$ values for lower AOI, i. e., $26^{\circ}$ and $34^{\circ}$. Nonetheless, at larger AOI, the increasing of wind speed results in an average reduction of $\sigma_{\text {CPD }}$ values (-28\%.) When dealing with the oil seep (Fig. 3(b)), no clear global trend is observed. The aforementioned sensitivity analysis suggests that the impact of AOI in broadening the CPD distribution is more significant that the one due to wind speed, and it is more pronounced over sea surface.

In the second experiment the slick-free and oil seep VV backscattering $\left(\sigma_{V V}^{0}\right)$ values were evaluated randomly sampling 1000 pixels belong to the ROIs. For each SAR dataset portioned according the AOI, it was evaluated the mean $\sigma_{V V}^{0}$ and the NESZ. Then, the mean signal to noise ratio (SNR) was calculated for each subset (see Table 1). Deal with sea surface, the SNR is well-above the $3 \mathrm{~dB}$ threshold for low and intermediate AOI; while, for the high AOI, the SNR goes below the threshold. For the oil seep, the SNR is well-above the threshold considering the low AOI; however, considering the intermediate and high AOI, the SNR is very close to or also goes below the threshold, respectively. This results in a larger depolarization, i. e., broader CPD distribution, due to noise rather than the surface properties. Accordingly, noise can concur in an enhanced sea oil seep detection, while reliable sea oil seep characterization is possible only at low AOI. The third experiment is designed to assess the extra-benefits provided by the polarimetric information for oil seep monitoring purposes. A pair of SAR scenes acquired with less than 24 hours time lag between each other is excerpted from the whole dataset (see Fig.4). An unsupervised 3-class $k$-means clustering algorithm is applied on VV-polarized intensity and CPD standard deviation images shown in Fig. 4(a)-(d). The results are shown in Fig. 4(e)-(h), respectively. Considering the $k$-means clustering based on the intensity information (Fig. 4(e)-(f)), over the slick-free sea area a heterogeneous patch is observed where the green color (intermediate intensity values) dominates; however there are few isolated clusters coded in blue and red color (lower and larger intensity values, respectively). This is likely due to the variability of sea surface roughness induced by winds, currents, etc. and to the surface occurrence of minor oil seeps. Over the sea area affected by the oil seep, an almost homogeneous region is clustered in blue color.

When dealing with the $k$-means clustering based on the $\sigma_{\mathrm{CPD}}$ (Fig. 4(g)-(h)), the slick-free sea surface is dominated by red color (lower $\sigma_{\mathrm{CPD}}$ values), as expected; even though few spots coded in green color (intermediate $\sigma_{\mathrm{CPD}}$ values) are observed. The oiled sea surface is dominated by blue color (largest $\sigma_{\mathrm{CPD}}$ 
values), as expected due to its strong damping properties that decorrelate the co-polarized channels, with some green spots located along the slick borders directly exposed to the wind action. Since larger values of $\sigma_{\mathrm{CPD}}$ results from stronger oil damping properties [6], those intermediate values are likely related to the oil layer more affected by aging and weathering phenomena. Hence, this experiment suggests that the copolarized phase information is able to offer key information on the spatial variability of the oil damping properties giving useful support to oil exploration and extraction activities which can not be provided from the intensity information.

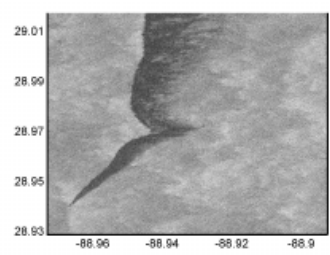

(a)

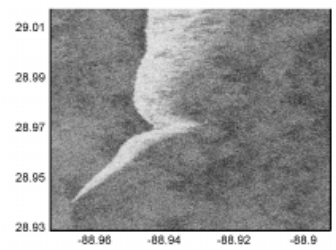

(c)

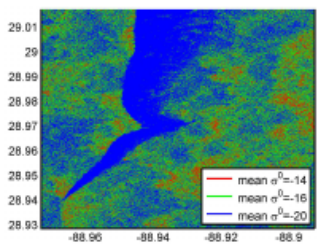

(e)

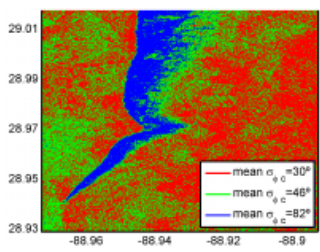

(g)

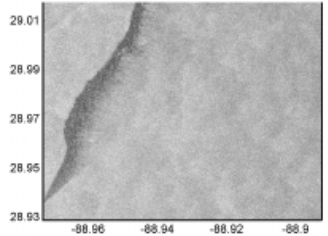

(b)

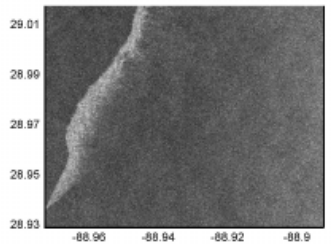

(d)

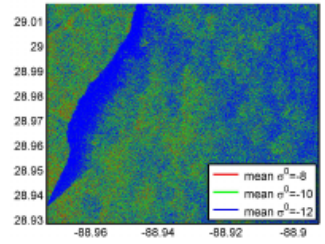

(f)

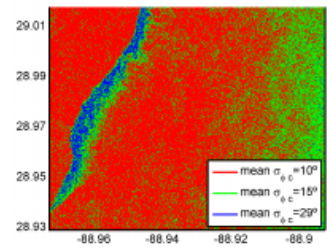

(h)
Fig. 4. Excerpts of TSX VV-polarized intensity ((a) and (b)), $\sigma_{\mathrm{CPD}}((\mathrm{c})$ and $(\mathrm{d}))$ images, and the corresponding $k$-means clustered outputs $((\mathrm{e})-(\mathrm{h}))$. Columns refer to SAR scenes acquired on $27 / 10 / 2013$ at $\mathrm{AOI}=34^{\circ}$ and on 28/10/2013 at $\mathrm{AOI}=26^{\circ}$, respectively.

\section{CONCLUSIONS}

A large time series of HH-VV TSX SAR scenes collected over a well-known oil seep in Gulf of Mexico is exploited for sea oil seep observation purposes. The main outcomes are: i) AOI, SNR and wind speed have an impact on the CPD distribution over both slick-free and slick-covered sea surface, that becomes broader when AOI increases and SNR descreases and narrower when wind speed increases, globally; and ii) the co-polarized channels phase information offers unprecedented benefits in mapping the oil seep damping properties.

\section{ACKNOWLEDGMENTS}

We thank the German Space Agency (DLR) for providing the TSX SAR data (ID AO OCE1045), and the National Oceanic and Atmospheric Administration (NOAA) for providing free buoy data.

\section{REFERENCES}

[1] N. A. D'souza, A. Subramaniam, A. R. Juhl, M. Hafez, A. Chekalyuk, S. Phan, B. Yan, I. MacDonald, S. Weber, and J. Montoya, "Elevated surface chlorophyll associated with natural oil seeps in the gulf of mexico," $\mathrm{Na}$ ture Geosci., vol. 9, no. 3, pp. 215-218, 2016.

[2] M. J. Collins, M. Denbina, B. Minchew, C. E. Jones, and B. Holt, "On the use of simulated airborne compact polarimetric sar for characterizing oil-water mixing of the deepwater horizon oil spill," IEEE J. Sel. Topics Appl. Earth Observ. Remote Sens., vol. 8, no. 3, pp. 1062-1077, 2015.

[3] M. Migliaccio, F. Nunziata, and A. Buono, "Sar polarimetry for sea oil slick observation," Int. J. Remote Sens., vol. 36, no. 12, pp. 3243-3273, 2015.

[4] G. Suresh, C. Melsheimer, J.-H. Körber, and G. Bohrmann, "Automatic estimation of oil seep locations in synthetic aperture radar images," IEEE Trans. Geosci. Remote Sens., vol. 53, no. 8, pp. 4218-4230, 2015.

[5] R. Jatiault, D. Dhont, L. Loncke, and D. Dubucq, "Monitoring of natural oil seepage in the lower congo basin using sar observations," Remote Sens. Env., vol. 191, pp. 258-272, 2017.

[6] M. Migliaccio, F. Nunziata, and A. Gambardella, "On the co-polarized phase difference for oil spill observation," Int. J. Remote Sens., vol. 30, no. 6, pp. 1587-1602, 2009.

[7] I. Hajnsek, E. Pottier, and S. R. Cloude, "Inversion of surface parameters from polarimetric sar," IEEE Trans. Geosci. Remote Sens., vol. 41, no. 4, pp. 727-744, 2003.

[8] A. Buono, F. Nunziata, and M. Migliaccio, "Analysis of full and compact polarimetric sar features over the sea surface," IEEE Geosci. Remote Sens. Lett., vol. 13, no. 10, pp. 1527-1531, 2016. 